\title{
The long and winding road of digital distribution. Or: why the ECJ's UsedSoft decision is of no use to keysellers
}

\author{
Dr Andreas Lober, ${ }^{*}$ Susanne Klein, LL.M. ${ }^{* *}$ and Florian Groothuis ${ }^{* \star *}$
}

\begin{abstract}
The 2012 UsedSoft decision of the Court of Justice of the European Union ('ECJ') disrupted the digital distribution of computer programs. Since then, unauthorised sellers of product and game keys often try to utilise the UsedSoft ruling and its principles to justify the resale of key codes. Against this background, we will review the new developments in this area, taking into account recent court decisions in Germany, which are based on European Directives. Furthermore, the impact of the UsedSoft decision on the gaming sector, where game keys are often distributed in digital form only, will be examined. Since the ECJ in its Nintendo decision and the German Federal Supreme Court 'BGH') in its World of Warcraft I decision both considered video games to be hybrid products, we will discuss whether and to what extent the Software Directive 2009/24/EC and the InfoSoc Directive 2001/29/EC are applicable. We then apply the findings to the distribution models used in the gaming sector and examine their legality.
\end{abstract}

Keywords: keyselling, game key, exhaustion, first sale, distribution, reproduction, hybrid products, UsedSoft, Nintendo, dual protection

\section{Introduction}

\subsection{The business model of selling video game key codes}

'AAA blockbuster games for an amazing EUR 35 - EUR 20 off the RRP for Western Europe': such offers abound on various websites selling stand-alone download keys (or 'game keys'). The origins of these game keys are manifold. Some might be taken out of the box of a retail product, or from a bundle (maybe with a new console), some stem from digital online distribution, and some were intended for a prize draw or other promotional measure. The reasons for such low prices are equally diverse. A games publisher may have granted high discounts to distributors and high street retailers, in order to have the games in stores nationwide. Instead of distributing the games as intended, some of these retailers might find it easier to scratch the keys, and sell them to keyselling websites. For online distribution, some game publishers try to maintain comparatively high prices for rich regions (e.g. North America and the EU), while offering the same game at lower prices in

Rechtsanwalt (Attorney at Law), Partner, Beiten Burkhardt, Frankfurt (Andreas.Lober@bblaw.com). other regions. These high prices are necessary to recoup ever-increasing development costs, but are understandably not very popular with players. Some of the big platforms selling game keys may have even developed schemes to escape VAT - whether or not such schemes are legal is a subject for a different paper. It also seems that some of these sites are far from compliant with customer protection laws, data protection laws, youth protection laws, or even gambling laws - which is also a way to undercut prices.

As a consequence of the multi-language policy of some game publishers, such as Steam, a game key purchased in Russia, for example, generally gives access not only to the Russian language version, but to all available language versions of a game, so that there are no de facto barriers for trading game keys. Regional locks could prevent cross-border sales, but the European Commission recently opened proceedings against several games publishers which have implemented such locks. Geo-blocking is therefore not a safe option at this time, at least not when it creates trade barriers between the Member States of the EU.

\footnotetext{
** Rechtsanwältin (Attorney at Law), Partner, Beiten Burkhardt, Frankfurt (Susanne.Klein@bblaw.com).

*** Research Assistant, Beiten Burkhardt, Frankfurt.
} 
Yet wasn't there something in copyright law, which gives right holders control over the distribution of their content? Well, there was - at least before the ruling of the Court of Justice of the European Union ('ECJ') in UsedSoft, ${ }^{1}$ which has officially legalised the reselling of used computer software.

This article will show that platforms selling game keys without the appropriate authorisation from the rights holder ('keysellers') are violating copyright law in Germany, and elsewhere in Europe. The ECJ's UsedSoft decision is of no use to them.

\subsection{Keyseller and copyright holder $-\mathrm{a}$ conflict of interest}

Now, let's get serious.

Authors of a work protected under copyright law generally have an interest in receiving an appropriate reward for previous investments ${ }^{2}$ and in return for their contribution to economic and cultural developments. ${ }^{3}$ This interest is acknowledged inter alia by Article 14(1)(i) of the Revised Berne Convention, which grants the copyright holder an exclusive distribution right regarding their work. It consists of a positive right to decide how the work shall be distributed and a negative right to prohibit the resale by third parties.

However, the copyright holder's distribution right is not absolute, but is restricted by the doctrine of exhaustion, which is also laid down in Article 6(2) of the WIPO Copyright Treaty and is known in the US as the 'first sale doctrine'. The exhaustion of the copyright holder's distribution right after the first sale of their work is designed to facilitate the free movement of goods and avoid the partitioning of markets. The copyright holder's distribution right therefore needs to be limited to the extent that is necessary to safeguard intellectual property. ${ }^{4}$

Since advocates of the keyseller business model refer from time to time to the doctrine of exhaustion in order to legitimise the resale of game keys, it is necessary to examine its legal scope and determine to what extent it restricts the copyright holder's distribution right.

\section{Regulatory framework in the European Union}

Within the EU, there are two directives that are particularly pertinent to our discussion: the Information Society Directive (Directive 2001/29/EC, 'InfoSoc Directive') and the Software Directive (Directive 2009/24/EC, 'Software Directive ${ }^{5}$ ).

The InfoSoc Directive has a broad scope of application because it is intended to ensure adequate protection for copyrighted works and related rights in the information society. ${ }^{6}$ In particular, creative and artistic works incorporated into a physical medium fall under the protective regime of the InfoSoc Directive. Computer programs, on the other hand, do not fall within the scope of the InfoSoc Directive as, according to Article 1(2)(a) of the Directive, they are addressed by the more specific Software Directive.

How these two directives interact in detail is a source of endless joy for masochistic academics, less so for practitioners or laymen. Both directives grant substantially different levels of protection for computer programs and other copyright protected works.

For the purpose of our discussion, the major difference between the two directives is the extent to which the copyright holder's exclusive distribution right ${ }^{7}$ is restricted by the doctrine of exhaustion. This was the main issue addressed by the UsedSoft ruling of the ECJ, which we will discuss directly.

(Another major difference between the two directives is the implementation of technological measures to prevent the unauthorised alteration or distribution of works through circumvention methods. This is not the subject of our discussion, but is dealt with in court decisions that we will mention later. At this point let us just note that Article 6 of the InfoSoc Directive acknowledges the implementation of technological protection measures and obliges Member States to implement an adequate legal protection regime in order to safeguard such measures. In contrast, the Software Directive does not predefine technological measures for protection against possible circumvention by third parties. It merely requires the implementation of appropriate remedies for any acts of putting infringing copies of a computer program into circulation. ${ }^{8}$ )
1 Case C-128/11 UsedSoft GmbH v. Oracle Int'l Corp. [2012] ECR I-0000, ('UsedSoft').

2 Recital 10 of Directive 2001/29/EC of the European Parliament and of the Council of 22 May 2001 on the harmonisation of certain aspects of copyright and related rights in the information society (OJ 2001 L 167, p. 10) ('InfoSoc Directive').

3 'World Economic Forum, Global Agenda Council on Intellectual Property System - Digital Copyright Principles', http://www3.weforum.org/docs/ WEF_GAC_CopyrightPrinciples.pdf, accessed 15 January 2018.
4 Case C-200/96 Metronome Musik v. Music Point Hokamp [1998] ECR I-1953, para 14.

5 Directive 2009/24/EC of the European Parliament and of the Council of 23 April 2009 on the legal protection of computer programs (OJ 2009 L 111, p. 16).

6 Recital (12) of the InfoSoc Directive.

7 Art 2 of the InfoSoc Directive; Art 4(1)(a) of the Software Directive.

8 Art 7 of the Software Directive. 


\section{Reselling 'used' software legalised by the UsedSoft decision}

\subsection{Facts of the UsedSoft case}

The German Federal Supreme Court (Bundesgerichtshof, ' $B G H$ ') asked the ECJ for a preliminary ruling on the resale of 'used' software. The software company Oracle developed computer software and distributed copies of that software to customers via the company's website. Customers purchasing the software program were allowed to download the 'client-server-software' from the website. Oracle only offered customers group licences with a minimum of 25 users each and granted a perpetual right to use in its licence agreement. Thus, companies who needed fewer than 25 user licences still had to purchase a group licence for 25 users. UsedSoft's business model was based on acquiring excess licences from such Oracle customers. After UsedSoft acquired those 'used' software licences, it resold them to its own customers and enabled those customers to download the client-server-software from the Oracle website. ${ }^{9}$

\subsection{Equality of physical and digital media under the Software Directive}

Prior to the ECJ's decision, there was a dispute as to whether distribution rights can exhaust only with regards to a copy of a computer program incorporated into a physical medium, or whether they are also exhausted when the computer program is the subject of digital medium. The ECJ took an economic point of view and stressed the similarity of selling a computer program on a physical medium, such as a DVD, and selling it as a download from the internet. It concluded that the online transmission of a computer program should be seen as functionally equivalent to the supply of a physical medium. ${ }^{10}$ Furthermore, the ECJ interpreted the exhaustion of the distribution right in the light of the principle of equality and considered it irrelevant whether a copy of a computer program is supplied on a physical medium to the first acquirer or whether he or she downloads it from the copyright holder's website. ${ }^{11}$ Accordingly, exhaustion can occur to both, physical and digital copies of a computer program.

9 Case C-128/11 UsedSoft GmbH v. Oracle Int'l Corp. [2012] ECR I-0000, paras 20-26.

10 Case C-128/11 UsedSoft GmbH v. Oracle Int'l Corp. [2012] ECR I-0000, para 60.

11 Case C-128/11 UsedSoft GmbH v. Oracle Int'l Corp. [2012] ECR I-0000, para 47.

12 The EEA includes all of the EU Member States as well as Iceland, Liechtenstein and Norway.

\subsection{Exhaustion criteria under the Software Directive}

The ECJ established certain criteria for determining the conditions under which the copyright holder's distribution rights are exhausted. The first sale of a copy of the computer program by the copyright holder or with their consent must have taken place on a market within the European Economic Area ${ }^{12}$ ('EEA'). If the first sale takes place outside of the EEA, the doctrine of exhaustion will not apply and the resale of a copy of the computer program will always violate the copyright holder's distribution right unless properly authorised. (An internationally applicable doctrine of exhaustion has not yet been recognised. ${ }^{13}$ )

The ECJ defines a 'first sale' as the transfer of a copy of the computer program by the copyright holder to the first acquirer which is accompanied by a user licence agreement. In this regard, the 'first sale' does not refer solely to the conclusion of a contract between the parties, but to the transfer of the physical or digital medium from the seller to the customer as well. ${ }^{14}$ Furthermore, the copyright holder must have granted the first acquirer a permanent licence and should have received an appropriate payment in return.

When these criteria are fulfilled, the copyright holder's distribution right is exhausted, and the first acquirer may resell a copy of a computer program without violating the distribution right. However, the first acquirer must take further precautions when reselling computer programs in order not to violate the copyright holder's exclusive right, pursuant to Article 4(1)(a) of the Software Directive, to reproduce the work, as the reproduction right is not subject to exhaustion. For this, the first acquirer must make their own copy unusable at the time of resale. Only under these conditions will the first and all subsequent acquirers be recognised as a 'lawful acquirer' within the meaning of Article 5(1) of the Software Directive. ${ }^{15}$

Thus, in its UsedSoft decision the ECJ recognised the applicability of the exhaustion doctrine in Article 4(2) of the Software Directive to a copy of a computer program that is distributed online and can be downloaded through the internet and stored on a data carrier.

13 Case C-479/04 Laserdisken ApS v. Kulturministeriet [2006] ECDR 30, para 27.

14 Case C-128/11 UsedSoft GmbH v. Oracle Int'l Corp. [2012] ECR I-0000, para 48.

15 Case C-128/11 UsedSoft GmbH v. Oracle Int'l Corp. [2012] ECR I-0000, para 85 . 


\section{Recent case law regarding keyselling of download keys for computer software}

The UsedSoft decision does not give keysellers a carte blanche to resell product keys. The ECJ established high hurdles in its UsedSoft ruling that must be overcome in order to ensure that the copyright holder's exclusive rights are not infringed. Recently, three German courts - the BGH, and the Higher Regional Courts of Frankfurt and Munich - had to decide on the sale of keys for computer software and the high hurdles established in the UsedSoft ruling.

\subsection{BGH - Exhaustion not dependent on form of distribution}

Although the BGH has not dealt with the isolated resale of game keys so far, it did deal with product keys for a computer program in its Green-IT decision. ${ }^{16}$

In the light of the principles set by the ECJ in the UsedSoft decision with respect to the Software Directive, the BGH applied the principle of equality and considered the sale of a computer program on a physical medium, such as a DVD, and the sale of a program by download from an online platform as similar. ${ }^{17}$ By acknowledging both forms of distribution as functionally equivalent, the Court considered the respective copy of the computer program as decisive for the application of the exhaustion doctrine, not the chosen form of distribution. ${ }^{18}$ The BGH concluded that it is irrelevant for the question of applicability of the exhaustion doctrine that a computer program had been sold for the first time as a box version and the product key is subsequently sold separate from the DVD. Thus, the exhaustion doctrine extends to the resale of the computer program by making the product key of the respective box version public.

\subsection{Higher Regional Court of Frankfurt \\ 4.2.1. Transmission of a non-activated product key is not distribution}

In 2016, the Higher Regional Court of Frankfurt am Main dealt with the sale of non-activated product keys for a computer program. ${ }^{19}$ While the UsedSoft decision dealt with volume licences that had already been activated by the

6 BGH NJW 2015, 3576

17 BGH NJW 2015, 3576, at 3579.

18 BGH NJW 2015, 3576, at 3579.

19 OLG Frankfurt a.M., 6 W 42/16 - short form in MMR 2016, 819, at 820.

20 Case C-128/11 UsedSoft GmbH v. Oracle Int'l Corp. [2012] ECR I-0000, paras 70,78 . original acquirer and were sold as 'used' software to subsequent acquirers, ${ }^{20}$ it is also common for licences to be sold for use on a computer by a single user. Product keys that enable the user to download a copy of a computer program only once are sold without prior activation.

(Once a product key is activated, the respective computer program may be linked to an account on an online distribution platform and cannot be used again to activate it for another account. This is especially common in the games sector, where a game is generally linked e.g. to a Steam account after the game key has been entered. The BGH considered this to be compatible with the exhaustion doctrine in its Half Life 2 decision, ${ }^{21}$ even though such business models make the sale of 'used' games impossible.)

The Frankfurt Court found that a product key, which has not been activated at the time of resale, does not serve to distribute a digital copy of a computer program since such distribution requires an already existing copy.

Instead, the Court stated that a non-activated product key serves the purpose of reproducing a copy of a computer program. Thus, in the absence of an existing copy, no 'used' software was being transferred and, consequently, neither the doctrine of exhaustion nor the principles laid down in the UsedSoft decision apply in this case. ${ }^{22}$ This means that the keyseller does not infringe the copyright holder's exclusive distribution right in such case, but may possibly infringe their reproduction right. ${ }^{23}$

\subsubsection{Transmission of a product key can be a reproduction}

As indicated above, the copyright holder's reproduction right pursuant to Article 4(1)(a) of the Software Directive may be infringed when the subsequent acquirer activates the purchased product key and produces a copy of the computer program by downloading it onto a local data carrier, unless the rights holder agrees to such reproduction.

\subsection{Higher Regional Court of Munich \\ 4.3.1. Transmission of a product key is distribution of the computer program}

The Higher Regional Court of Munich recently considered the transmission of an activated product key and a link for

21 BGH MMR 2010, 771, at 773.

22 OLG Frankfurt a.M. MMR 2016, 819, at 820.

23 T. Stögmüller 'Weiterverkauf eines Produktschlüssels vor Download' (2016) GRUR-Prax 337. 
downloading a copy of the computer program as a distribution of a computer program. ${ }^{24}$ This means that the keyseller must be able to prove the origin of the product keys, and that the copyright holder has received appropriate payment. ${ }^{25}$ Without this evidence the copyright holder's exclusive distribution right will be infringed.

\subsubsection{Transmission of a product key and download link creates risk of a reproduction}

Furthermore, when a keyseller transmits a product key and a download link to the acquirer without being authorised to do so by the copyright holder, the keyseller enables the acquirer to download a copy of the computer program and install it on their own computer. This action establishes a risk that the acquirer will infringe the copyright holder's exclusive right of reproduction.

\subsubsection{Higher Regional Court of Munich: Licence key does not grant a licence - it is a technical measure, like the key of a car}

The common promotion of product keys as 'Lizenzschlüssel ${ }^{26}$ in Germany should not obscure the fact that product keys do not embody a licence, which allows the acquirer to use the respective computer program without the copyright holder's authorisation. Therefore, it remains necessary to obtain a licence for the respective computer program in order not to infringe the copyright holder's rights. The Higher Regional Court of Munich also confirmed this, comparing product keys to car keys, the mere function of which is to unlock the corresponding car. The product key's inherent function is that of a technical device, which enables the acquirer to activate the purchased computer program and use it to the extent allowed in the licence agreement. ${ }^{27}$

\subsubsection{Conclusion of the Higher Regional Court of Munich}

Only where the principles of the UsedSoft decision apply and the copyright holder's distribution right is exhausted can the transmission of the product key and download link be justified and the establishment of a risk for the reproduction right be avoided.

\subsection{Summary of the Court Judgments}

According to the Higher Regional Courts of Frankfurt and Munich, making product keys available will generally interfere with the right owner's reproduction and (at least for keys which have been activated) distribution rights. As far as computer software is concerned, this can only be legal if the original rights are exhausted which will not be the case for non-activated keys. Video game product keys are mostly non-activated as they are linked to game accounts, e.g. on Steam.

\section{Applying the findings in the UsedSoft judgment to video games}

Still, there is another reason why the principle of exhaustion does not help keysellers.

Applying the findings of the UsedSoft ruling and national court judgments based on it to video games would require video games to be qualified as computer programs. However, games are not merely code - it is the combination of story, music and graphic elements that makes a great game.

This is why we will now examine whether it is appropriate to view games as just computer programs.

\subsection{Video games - computer program, creative work, or both?}

It is difficult to find a legal definition of a computer program; the EU specifically did not define the term 'computer program' in the Software Directive in an effort to remain neutral towards future technical developments. Nevertheless, Section 1(i) of the WIPO Model Provisions on the Protection of Computer Software provides a useful assistance for understanding the key elements of a computer program. Accordingly, a computer program is 'a set of instructions capable, when incorporated in a machine-readable medium, of causing a machine having information-processing capabilities to indicate, perform or achieve a particular function, task or result'. Since the sequences of a video game are influenced by a control program that works according to this definition, video games are based on a computer program.

However, video games also contain graphic and sound elements. Audio-visual elements, the game's characters and its storyline attract gamers and, therefore, may be equally as important as the computer program itself.

A video game that does not have a computer program implemented would not be able to function, while a video game without audio-visual elements would not 
generate visual or audio feedback to allow the gamer to interact with the game.

Consequently, the ECJ acknowledged in its Nintendo v. PC Box judgment that video games are complex matter comprising not only the computer program but also graphic and sound elements, and, as such, the technical and artistic combination is part of a video game's originality and constitutes a unique creative value. ${ }^{28}$ In light of this ruling, the computer program and artistic elements are clearly both essential elements of a video game.

Recognising video games as hybrid product as prescribed in the Nintendo v. PC Box ruling ${ }^{29}$ inevitably leads to the issue of determining the applicable scope of protection for video games: do they fall under the Software Directive or the InfoSoc Directive? The determination of the applicable regime is decisive for the copyright holder's rights. ${ }^{30}$ In the context of the Software Directive, the distribution right can be exhausted with respect to both physical and digital media if the reseller meets the criteria established in the UsedSoft decision. Under the InfoSoc Directive, however, exhaustion is limited to works incorporated into physical media, not to works distributed in digital form only.

Accordingly, if video games are protected under the InfoSoc Directive, the principle of exhaustion cannot apply to game keys, which stem from digital distribution (as opposed to retail games). It is therefore important to take a closer look at the protection of hybrid products.

\subsection{Applicable protection regime for video games in the EU and Germany}

The ECJ and BGH were both faced with the question whether the Software Directive or the InfoSoc Directive is applicable to video games as hybrid products. Although their rulings did not specifically address the issue of the applicability of the exhaustion doctrine, they discussed the protection regime (Software Directive or InfoSoc Directive) and, therefore, their findings have a direct impact on the exhaustion regime as well.

\subsubsection{ECJ - Nintendo v. PC Box judgment}

The ECJ had to decide in its Nintendo ruling - which concerned devices that circumvented technological protection measures - whether video games are protected under the Software Directive or the InfoSoc Directive. This issue was decisive because the InfoSoc Directive allows the copyright holder to implement technological measures to protect their work against unauthorised acts to an extent that is not supported under the Software Directive. ${ }^{31}$ Since the InfoSoc Directive treats different parts of a work like the whole work, as long as they are protected by copyright, the ECJ assumed that the InfoSoc Directive is applicable when copyright protectable parts 'share the originality of the whole work' ${ }^{32}$ In this context, the ECJ considered graphic and sound elements as part of a game's originality. Therefore, the ECJ found that the audio-visual elements shall be protected 'together with the entire work' ${ }^{33}$ and applied the InfoSoc Directive to determine the scope of the applicable protection measures for video games. ${ }^{34}$

\subsubsection{BGH - World of Warcraft I decision}

The BGH also acknowledged the unique combination of programming and audio-visual elements of video games in its World of Warcraft I decision. ${ }^{35}$ This case concerned the production and distribution of cheat bots, which were not authorised by the games publisher. The producer of the bot claimed that it only observed, studied and tested the functioning of the video game in order to determine its concepts and principles and produce a bot. Accordingly, the bot producer argued, this would not require the copyright holder's authorisation pursuant to Section 69d(3) of the German Copyright Act. $^{36}$ The BGH did not follow the view of the botproducer and declared that Section 69d(3) of the German Copyright Act was not applicable because this section applies to computer programs only. The Court held that audio-visual elements of a video game fall under the protective regime of the InfoSoc Directive, which does not contain an exemption clause similar to that of Article 5(3) of the Software Directive. ${ }^{37}$
28 Case C-355/12 Nintendo Co. Ltd v. PC Box Srl. [2014] ECDR 6, para 23.

29 Case C-355/12 Nintendo Co. Ltd v. PC Box Srl. [2014] ECDR 6, para 23; Austria took the same approach, see OGH, ZUM-RD 2005, 11, at 14 (Oberster Gerichtshof, Supreme Court of Justice of the Republic of Austria).

30 For cases where the parallel applicability would lead to inconsistent results, see W. Bullinger and C. Czychowski, 'Digitale Inhalte: Werk und/oder Software' (2011) GRUR 19, 24

31 Arts 5 and 6 of the Software Directive determine numerous exemptions for cases where protection measures are not allowed to be implemented.

32 Case C-355/12 Nintendo Co. Ltd v. PC Box Srl. [2014] ECDR 6, para 22.

33 Case C-355/12 Nintendo Co. Ltd v. PC Box Srl. [2014] ECDR 6, para 22.
34 Belgium, Brazil, Denmark, Egypt, France, India, Japan, South Africa and Sweden separate the work elements of a video game and apply protection individually, see A. Ramos, L. López, A. Rodriguez, T. Meng and S. Abrams 'The Legal Status of Video Games: Comparative Analysis in National Approaches' [2013] WIPO para 14, http://www.wipo.int/ export/sites/www/copyright/en/activities/pdf/comparative_analysis_on_ video_games.pdf, last accessed 22 January 2018.

35 BGH GRUR 2017, 266, at 273.

36 The norm in Art 5(3) of the Software Directive was transposed into German law through the German Copyright Act.

37 BGH GRUR 2017, 266, at 273. 


\subsubsection{Relevance for keyselling}

While neither of these Courts had to decide on keyselling, or even on the applicability of the exhaustion doctrine, ${ }^{38}$ both judgments provide some guidance: there can only be exhaustion for game keys when the criteria set forth in the InfoSoc Directive are met - the first sale must not be digital.

\section{Exhaustion for video game key codes}

Next we will examine the impact of the exhaustion doctrine on different keyselling business models for video games. For this purpose, it will be assumed that the exhaustion doctrine under Article 4(2) of the Software Directive does not apply to video games as they are hybrid products; Article 4(2) of InfoSoc Directive applies instead. ${ }^{39}$

\subsection{Resale of digital key codes}

The most common form of distribution in the gaming sector is digital distribution via game keys, which allow the acquirer to download and install the video game software. As long as the video game has been sold in digital form only, no physical medium is transferred to change the ownership of the video game. Subsequently, the copyright holder's exclusive distribution right cannot be exhausted according to Article 4(2) of the InfoSoc Directive, the InfoSoc Directive as does not recognise the exhaustion of distribution rights for digital media. Therefore, any unauthorised distribution made by a keyseller would constitute a violation of the right holder's exclusive distribution right.

\subsection{DVD and printed product key combined in a box}

Another way to distribute a video game is to introduce a box version of the video game on a market within the EEA that includes, at least in theory, a copy of the video game software on a DVD and a printed product key. (This is a dying species - more often than not, the DVD only contains a small part of the game, and the remainder is downloaded during the first installation.)

Still, for the sake of completeness (and because there is an interesting court decision on the issue), let's assume

38 C Czychowski, 'Der BGH und Computerspiele: Es verbleiben noch offene Fragen' (2017) GRUR 362, 363.

39 However, it was predicted that the UsedSoft ruling would have a much broader impact than merely software, see E. Linklater, 'UsedSoft and the Big Bang Theory: Is the e-Exhaustion Meteor about to Strike' (2014) 5 Intell. Prop. Info. Tech. \& Elec. Com. L. 12, at 19.

40 LG Berlin K\&R 2014, 445. that box versions of games are distributed physically, not digitally. This means that the consumer can either install the video game on their computer using the DVD and activate the game by entering the printed game key, or just use the game key to start the download process from a digital distribution platform. Either way, the consumer needs the game key to start playing. After the game key has been entered on the distribution platform, the video game is linked to the consumer's game account and the game key cannot be used again. Keysellers are taking advantage of this form of distribution by purchasing box versions of video games on a market where they are offered at a comparably low price and selling the game keys without the DVD on a market with a comparably high price.

The Berlin District Court is the only court in Germany that has ruled in a published decision on the question of whether the keyseller can rely on the exhaustion of the copyright holder's distribution right when reselling the game key separately from the DVD, and it came to an interesting conclusion. ${ }^{40}$

Not surprisingly, the Court considered the resale of game keys as infringement of the copyright holder's exclusive distribution right unless it has been exhausted under the InfoSoc Directive. Since exhaustion can occur only when the work was introduced onto a market with the copyright holder's authorisation, the Court focused on the scope of the given authorisation. It concluded that when the copyright holder sells a video game for the first time as a combination package (i.e. a box containing a DVD and a game key), the authorisation for a resale is limited to the product as a combination package. ${ }^{41}$ By selling the game key to subsequent acquirers without the DVD, the keyseller departs from the product that has been subject of the first sale and is not authorised in the creation of a new product. ${ }^{42}$ Without permission to create a new product, the copyright holder's distribution right cannot be exhausted regarding game keys that are sold separately from the DVD of a combination package. ${ }^{43}$

\section{Conclusion}

In the light of all this, keysellers of game keys cannot sell game keys online that have been introduced by the copyright holder digitally within the EEA, nor can they

41 LG Berlin K\&R 2014, 445, at 446.

42 S. Telle, 'K\&R-Kommentar' (2014) K\&R 445, at 447.

43 L.G. Berlin K\&R 2014, 445, at 446; see also F. Hilgert, 'Keys und Accounts beim Computerspielvertrieb' [2014] CR 354, at 357 and C Wolff 'Urheberrechtswidriges Keyselling' (2014) ITBR 155, at 156 
separately sell game keys that were part of a box version and combined with a physical medium (DVD) without infringing the copyright holder's exclusive rights.

Video games are hybrid products and fall under the protection regime of the InfoSoc Directive (see the ECJ Nintendo and BGH World of Warcraft judgments). Thus, exhaustion cannot occur to games when the first acquirer purchased them through digital distribution channels.

Under the InfoSoc Directive, therefore, it is only really worth discussing exhaustion for retail games. However, retail games are rarely distributed physically now - in general, a major part of the game has to be downloaded by activating it through the game key. Until this has happened, the copyright holder's rights have not been exhausted, as the product has not been fully brought onto the market (Higher Regional Court Frankfurt). Non-activated game keys, therefore, cannot exhaust the copyright holder's right (and activated game keys are linked to a game account and cannot be resold). Furthermore, splitting up a retail combination product and distributing the game key separately cannot be justified by the principle of exhaustion (Regional Court Berlin). Finally, it might be argued that selling game keys separately from the DVD is a circumvention of technological protection measures. 